\title{
Estimating and Forecasting with a Dynamic Spatial Panel Data Model
}

Badi H Baltagi (Syracuse University, Center for Policy Research) Bernard Fingleton (SERC, SIRE, University of Strathclyde)

Alain Pirotte (ERMES (CNRS), Université Panthéon-Assas Paris II/Sorbonne université, IFSTTAR-DEST)

November 2011 
This work is part of the research programme of the independent UK Spatial Economics Research Centre funded by the Economic and Social Research Council (ESRC), Department for Business, Innovation and Skills (BIS), the Department for Communities and Local Government (CLG), and the Welsh Assembly Government. The support of the funders is acknowledged. The views expressed are those of the authors and do not represent the views of the funders.

(C) B. H. Baltagi, B. Fingleton and A. Pirotte, submitted 2011 


\title{
Estimating and Forecasting with a Dynamic Spatial Panel Data Model
}

\author{
Badi H. Baltagi* \\ Bernard Fingleton** \\ Alain Pirotte***
}

November 2011

* Syracuse University, Center for Policy Research

** SERC, Department of Land Economy, Cambridge University

*** ERMES (CNRS), Université Panthéon-Assas Paris II/Sorbonne universités, IFSTTARDEST

Acknowledgements

This paper was presented at the Economics Seminar Series, Strathclyde University, Glasgow, Scotland, March 30, 2011. Also, at the Vth Conference of the Spatial Econometrics Association, Toulouse University, France, July 6-8, 2011. We would like to thank Patrick Sevestre and the participants of this seminar and this conference for their useful comments and suggestions. 


\begin{abstract}
$\underline{\text { Abstract }}$
This paper focuses on the estimation and predictive performance of several estimators for the dynamic and autoregressive spatial lag panel data model with spatially correlated disturbances. In the spirit of Arellano and Bond (1991) and Mutl (2006), a dynamic spatial GMM estimator is proposed based on Kapoor, Kelejian and Prucha (2007) for the Spatial AutoRegressive (SAR) error model. The main idea is to mix non-spatial and spatial instruments to obtain consistent estimates of the parameters. Then, a linear predictor of this spatial dynamic model is derived. Using Monte Carlo simulations, we compare the performance of the GMM spatial estimator to that of spatial and non-spatial estimators and illustrate our approach with an application to new economic geography.
\end{abstract}

Keywords: Panel data; spatial lag; error components; linear predictor; GMM; spatial autocorrelation

JEL classifications: C33 


\section{Introduction}

This paper considers spatial panel data models in which there is variation across time and space involving simultaneous spatial (network) dependence together with dynamic interaction. Spatial dependence models are popular in regional science and urban economics, where the cross-sectional units are typically locations (cities, countries, regions) which are affected by common factors or spillover effects from neighboring locations. Forecasting studies using spatial panel data models are rare, and those involving forecasting with a dynamic component are almost absent from the literature. Recently, Baltagi and Pirotte (2010) showed that tests of hypotheses based on the usual panel data estimators that ignore spatial dependence can produce misleading inference.

For dynamic panel data models with no spatial autocorrelation, it is well known that the OLS estimator is biased, see Trognon (1978), and Sevestre and Trognon $(1983,1985)$. Also, the fixed effects estimator is biased, see Nickell (1981) and Kiviet (1995). Anderson and Hsiao (1981, 1982) proposed an IV estimator which is consistent. Subsequent developments focused on Generalized Method of Moments (GMM) estimators including Arellano and Bond (1991) and Blundell and Bond (1998) to mention just a few. See Blundell, Bond and Windmeijer (2000), Arellano and Honoré (2001), Hsiao (2003) and Baltagi (2008) for good reviews and a textbook treatment of this subject.

Spatial dependence models deal with spatial interaction and spatial heterogeneity (see Anselin (1988), LeSage and Pace (2009)). The structure of the spatial dependence can be related to location and distance, both in a geographic space as well as a more general economic or social network space, see Anselin, Le Gallo and Jayet (2008). Typically, cross-section dependence is modelled as proportional to some observable distance, see Anselin (1988), LeSage and Pace (2009), introduced through an endogenous spatial lag variable or via spatially correlated disturbances, or both. Combining cross-section dependence with autoregressive (temporal) dependence leads us to Elhorst (2005), who derives a Maximum Likelihood Estimator (MLE). Another way to estimate autoregressive models with spatial dependence is to extend the GMM approach to the spatial case in order to obtain consistent parameter estimates. Jacobs, Ligthart and Vrijburg (2009) focus on a dynamic autoregressive fixed effects model which includes the spatial lag of the dependent variable together with spatially correlated disturbances. 
They propose a three-step GMM approach. Elhorst (2010) considers the same model except that the disturbances are not spatially autocorrelated. He develops Bias Corrected Least Squares Dummy Variables (BCLSDV), unconditional ML and GMM estimators. Védrine and Bouayad-Agha (2010) use a GMM spatial estimator, with similar orthogonality conditions to those of Arellano and Bond (1991), to study the convergence of European regions. Mutl (2006) extends the Kapoor, Kelejian and Prucha (2007) approach to a dynamic model with spatially correlated disturbances under less restrictive assumptions. Yu, de Jong and Lee (2008) propose a Quasi Maximum Likelihood Estimator (QMLE) for spatial dynamic panel data with fixed effects when both $N$ and $T$ are large. Lee and $\mathrm{Yu}(2010 a, b)$ extend this approach under different assumptions about $N$ and T. Kukenova and Monteiro (2009) consider a system-GMM to estimate a dynamic spatial panel model (i.e. first order spatial autoregressive panel data model). They compare its properties to those of the usual estimators (MLE, QMLE, LSDV, etc.).

In this paper, we propose a spatial GMM estimator in the spirit of Arellano and Bond (1991) and Mutl (2006) under the assumptions that the model includes temporal and spatial lags on the endogenous variable together with SAR-RE disturbances. Only a few articles study the predictive performance of spatial panel models. Baltagi and Li (2006), Baltagi, Bresson and Pirotte (2010), Fingleton $(2009,2010)$ focus on the particular case of a static model under spatially correlated disturbances. In contrast, Longhi and Nijkamp (2007), Kholodilin, Siliverstovs and Kooths (2008) use dynamic spatial models. Longhi and Nijkamp (2007) compare different models designed to compute short-term ex post forecasts of regional employment in a panel of 326 West German regions observed over the period from 1987 to 2002. They show that forecasts can be improved by simply taking into account the distances across regions. Nevertheless, this study is specific to the area and variables under investigation. Kholodilin, Siliverstovs and Kooths (2008) focus on multi-step forecasts of the annual growth rates of real GDP for each of the 16 German Länder simultaneously over the period from 2002 to 2006 (estimation period is 1993-2001). They do not consider any explanatory variables. Moreover, the forecasts are computed using reduced forms and ML estimates of the parameters. In this article, we present a GMM spatial procedure and derive a Linear Predictor (LP) for the more general case of a spatial dynamic model (i.e. one that includes a spatial lag together with spatially correlated disturbances). Using Monte Carlo simulations and an empirical illustration, we compare the empirical performance of our GMM spatial esti- 
mator to that of OLS, Within and GMM, each of which takes no account of the spatial structure of the disturbances. We also compare our spatial estimator to other spatial GMM estimators, such as Mutl (2006). Last, we also evaluate the predictive performance of our dynamic spatial model. The plan of the paper is as follows: Section 2 presents the model, section 3 focuses on our spatial GMM estimators. Section 4 derives a Linear Predictor. Section 5 describes the Monte Carlo design. Section 6 presents the results, Section 7 illustrates our approach using an application to new economic geography, and the last section concludes.

\section{The Spatial Dynamic Panel Model}

Consider a first order spatial autoregressive panel data model

$$
y_{i t}=\gamma y_{i t-1}+\rho_{1} \sum_{j=1}^{N} w_{i j} y_{j t}+x_{i t} \beta+\varepsilon_{i t} \quad i=1, \ldots, N ; t=1, \ldots, T,
$$

where $y_{i t}$ is the dependent variable for region $i$ at time $t, x_{i t}$ is a $(1 \times K)$ vector of explanatory (exogenous) variables, $\gamma$ and $\beta$ represent corresponding $(1 \times 1)$ and $(K \times 1)$ parameters to be estimate. $w_{i j}$ is the $(i, j)$ element of the matrix $W_{N}$. $W_{N}$ is an $(N \times N)$ known spatial weights matrix which has zero diagonal elements and is row-normalized. If $W_{N}$ is defined as first order contiguity, such elements consist of location pairs that have common border but there is no higher order contiguity. $\rho_{1}$ is the spatial lag coefficient. This parameter is assumed to be restricted to the interval $\left[1 / r_{\min }, 1\right]$, where $r_{\text {min }}$ equals the most negative purely real characteristic root of $W_{N}$ after this matrix is row-normalized, see LeSage and Pace (2009, pp. 88-89). This ensures stationarity across space. The stationarity assumption also requires $|\gamma|<1$. So, parameter combinations that violate $|\gamma|<1-\rho_{1}$ for $\rho_{1} \geq 0$ or $|\gamma|<1-\rho_{1} r_{\text {min }}$ for $\rho_{1}<0$ are excluded, otherwise the model is nonstationary in time. Moreover, in contrast to the usual panel data framework, we allow $\varepsilon_{i t}$ to be contemporaneously correlated according to the Spatial AutoRegressive (SAR) error model with row-normalized matrix $M_{N}$ with similar properties to $W_{N}$ but with elements $m_{i j} \neq w_{i j}$ :

$$
\varepsilon_{i t}=\rho_{2} \sum_{j=1}^{N} m_{i j} \varepsilon_{j t}+u_{i t},\left|\rho_{2}\right|<1
$$


The stationarity conditions imposed on $\rho_{2}$ are like those for $\rho_{1}$, in this case with respect to the real eigenvalues of $M_{N}$. Moreover, the remainder term $u_{i t}$ follows an error component structure

$$
u_{i t}=\mu_{i}+v_{i t}
$$

where $\mu_{i}$ is an individual specific time-invariant effect which is assumed to be $i i d\left(0, \sigma_{\mu}^{2}\right)$, and $v_{i t}$ is a remainder effect which is assumed to be $i i d\left(0, \sigma_{v}^{2}\right)$. $\mu_{i}$ and $v_{i t}$ are independent of each other and among themselves. Combining (2) and (3), we obtain the SAR-RE specification of the disturbance $\varepsilon_{i t}$. For simplicity, in practice, we assume that $M_{N}=W_{N}$.

\section{A Spatial GMM Estimator}

Following Anderson and Hsiao (1981, 1982), Arellano and Bond (1991), we eliminate the individual effect $\mu_{i}$ in (3), which is correlated with the lagged dependent variable, by differencing the model (1) yielding

$$
\Delta y_{i t}=\gamma \Delta y_{i t-1}+\rho_{1} \sum_{j=1}^{N} w_{i j} \Delta y_{j t}+\Delta x_{i t} \beta+\Delta \varepsilon_{i t} .
$$

In contrast to the classical literature on panel data, grouping the data by periods rather than units is more convenient when we consider the spatial correlation due to (2). For a cross-section $t$, we have:

$$
\Delta y_{t}=\gamma \Delta y_{t-1}+\rho_{1} W_{N} \Delta y_{t}+\Delta x_{t} \beta+\Delta \varepsilon_{t}
$$

where $y_{t}=\left(y_{1 t}, \ldots, y_{N t}\right)^{\prime}$ is a $(N \times 1)$ vector, and the matrix $x_{t}=\left(x_{1 t}, \ldots\right.$, $\left.x_{N t}\right)^{\prime}$ is of dimension $(N \times K)$. For the error vector $\varepsilon_{t}$ of dimension $(N \times 1)$, we assume $E\left(\varepsilon_{t}\right)=0$,

$$
E\left(\varepsilon_{t} \varepsilon_{t}^{\prime}\right)=\sigma_{\varepsilon}^{2}\left(B_{N}^{\prime} B_{N}\right)^{-1}
$$

where $\sigma_{\varepsilon}^{2}=\sigma_{\mu}^{2}+\sigma_{v}^{2}, B_{N}=\left(I_{N}-\rho_{2} W_{N}\right)$. The matrix $B_{N}$ is assumed to be nonsingular, and the row and column sums of the matrix $W_{N}$ are bounded uniformly in absolute value. The corresponding vector $(N(T-1) \times N(T-1))$ covariance matrix of $\varepsilon$ is given by

$$
\Omega=\left(\sigma_{\mu}^{2} J_{T-1}+\sigma_{v}^{2} I_{T-1}\right) \otimes\left(B_{N}^{\prime} B_{N}\right)^{-1}
$$


where $J_{T-1}$ is a $(T-1 \times T-1)$ matrix of ones, $I_{T-1}$, an identity matrix of order $T-1$.

Using the Arellano and Bond (1991) methodology, we can obtain a GMM estimator based on the following moment conditions:

$$
\begin{aligned}
E\left(y_{i l} \Delta v_{i t}\right) & =0 \quad \forall i, l=1,2, \ldots, T-2 ; t=3,4, \ldots, T \\
E\left(x_{k, i m} \Delta v_{i t}\right) & =0 \quad \forall i, k, m=1,2, \ldots, T ; t=3,4, \ldots, T
\end{aligned}
$$

where (9) assumes that the explanatory variables $x_{k, i m}$ are strictly exogenous. Moreover, we can use spatially dependent and explanatory variables as instruments. The validity of this strategy requires the following moments conditions:

$$
\begin{gathered}
E\left(\sum_{i \neq j} w_{i j} y_{j l} \Delta v_{i t}\right)=0 \quad l=1,2, \ldots, T-2 ; t=3,4, \ldots, T \\
E\left(\sum_{i \neq j} w_{i j} x_{k, j l} \Delta v_{i t}\right)=0 \quad \forall i, k, m=1,2, \ldots, T ; t=3,4, \ldots, T .
\end{gathered}
$$

Let us define the matrix $Z$ which contains the non-spatial instruments (i.e. related to the conditions (8) and (9)) as

$$
Z=\left(\begin{array}{cccc}
Z_{3} & 0 & \cdots & 0 \\
0 & Z_{4} & \ddots & \vdots \\
\vdots & \ddots & \ddots & 0 \\
0 & \cdots & 0 & Z_{T}
\end{array}\right)
$$

where

$$
Z_{t}=\left(y_{1}, \ldots, y_{t-2}, x_{1}, \ldots, x_{T}\right)
$$

is an $(N \times(t-2)+K T)$ matrix of instruments at time $t, y_{l}$ is a vector of dimension $(N \times 1)$ and $x_{r}$ is a matrix of dimension $(N \times K)$. Moreover, we can define a matrix $Z^{s}$ which contains the spatial instruments (i.e. related to the conditions (10) and (11)) as

$$
Z^{s}=\left(\begin{array}{cccc}
Z_{3}^{s} & 0 & \cdots & 0 \\
0 & Z_{4}^{s} & \ddots & \vdots \\
\vdots & \ddots & \ddots & 0 \\
0 & \cdots & 0 & Z_{T}^{s}
\end{array}\right)
$$


with

$$
Z_{t}^{s}=\left(y_{1}^{s}, \ldots, y_{t-2}^{s}, x_{1}^{s}, \ldots, x_{T}^{s}\right)
$$

where

$$
y_{l}^{s}=\left(\begin{array}{c}
\sum_{j=1}^{N} w_{1 j} y_{j l} \\
\sum_{j=1}^{N} w_{2 j} y_{j l} \\
\vdots \\
\sum_{j=1}^{N} w_{N j} y_{j l}
\end{array}\right)
$$

and

$$
x_{r}^{s}=\left(\begin{array}{cccc}
\sum_{j=1}^{N} w_{1 j} x_{1 j r} & \sum_{j=1}^{N} w_{1 j} x_{2 j r} & \cdots & \sum_{j=1}^{N} w_{1 j} x_{k j r} \\
\sum_{j=1}^{N} w_{2 j} x_{1 j r} & \sum_{j=1}^{N} w_{2 j} x_{2 j r} & \cdots & \sum_{j=1}^{N} w_{2 j} x_{k j r} \\
\vdots & \vdots & & \vdots \\
\sum_{j=1}^{N} w_{N j} x_{1 j r} & \sum_{j=1}^{N} w_{N j} x_{2 j r} & \cdots & \sum_{j=1}^{N} w_{N j} x_{k j r}
\end{array}\right) .
$$

If we stack the matrices $Z$ and $Z^{s}$, we obtain the valid instruments for the model (4), namely $Z^{*}$. Moreover, we use the weight matrix of moments

$$
A_{N}=\left[E\left[Z^{\prime}(\Delta \varepsilon)(\Delta \varepsilon)^{\prime} Z\right]\right]^{-1}
$$

with

$$
E\left[(\Delta \varepsilon)(\Delta \varepsilon)^{\prime}\right]=\sigma_{v}^{2}\left(I_{T-2} \otimes H_{N}\right)\left(G \otimes I_{N}\right)\left(I_{T-2} \otimes H_{N}^{\prime}\right)
$$

and

$$
G=\left(\begin{array}{cccccc}
2 & -1 & 0 & \cdots & 0 & 0 \\
-1 & 2 & -1 & \cdots & 0 & 0 \\
0 & -1 & \ddots & \ddots & \vdots & \vdots \\
\vdots & \ddots & \ddots & \ddots & -1 & 0 \\
0 & 0 & 0 & -1 & 2 & -1 \\
0 & 0 & 0 & \cdots & -1 & 2
\end{array}\right), H_{N}=B_{N}^{-1}=\left(I_{N}-\rho_{2} W_{N}\right)^{-1}
$$

A consistent estimate of the autoregressive parameter $\rho_{2}$ can be obtained using the Kapoor, Kelejian and Prucha (2007) approach, hereafter KKP. In fact, KKP generalized the GM procedure from cross-section data proposed by Kelejian and Prucha (1999) to panel data and derived its large sample properties when $T$ is fixed and $N \rightarrow \infty$. They proposed three generalized 
moments (GM) estimators of $\rho_{2}, \sigma_{v}^{2}$ and $\sigma_{1}^{2}\left(=\sigma_{v}^{2}+T \sigma_{\mu}^{2}\right)$ based on the following six moment conditions:

$$
E\left[\begin{array}{c}
\frac{1}{N(T-1)} u_{N}^{\prime} Q_{0, N} u_{N} \\
\frac{1}{N(T-1)} \bar{u}_{N}^{\prime} Q_{0, N} \bar{u}_{N} \\
\frac{1}{N(T-1)} \bar{u}_{N}^{\prime} Q_{0, N} u_{N} \\
\frac{1}{N} u_{N}^{\prime} Q_{1, N} u_{N} \\
\frac{1}{N} \bar{u}_{N}^{\prime} Q_{1, N} \bar{u}_{N} \\
\frac{1}{N} \bar{u}_{N}^{\prime} Q_{1, N} u_{N}
\end{array}\right]=\left[\begin{array}{c}
\sigma_{v}^{2} \\
\sigma_{v}^{2} \frac{1}{N} \operatorname{tr}\left(W_{N}^{\prime} W_{N}\right) \\
0 \\
\sigma_{1}^{2} \\
\sigma_{1}^{2} \frac{1}{N} \operatorname{tr}\left(W_{N}^{\prime} W_{N}\right) \\
0
\end{array}\right]
$$

where

$$
\begin{aligned}
& u_{N}=\varepsilon_{N}-\rho_{2} \bar{\varepsilon}_{N} \\
& \bar{u}_{N}=\bar{\varepsilon}_{N}-\rho_{2} \overline{\bar{\varepsilon}}_{N} \\
& \bar{\varepsilon}_{N}=\left(I_{T} \otimes W_{N}\right) \varepsilon_{N} \\
& \overline{\bar{\varepsilon}}_{N}=\left(I_{T} \otimes W_{N}\right) \bar{\varepsilon}_{N} .
\end{aligned}
$$

Following (21), (22) to (25), and if we consider the sample moments counterparts based on $\widetilde{\varepsilon}_{N}$, we can write:

$$
G_{N}\left[\rho_{2}, \rho_{2}^{2}, \sigma_{v}^{2}, \sigma_{1}^{2}\right]^{\prime}-g_{N}=\xi_{N}\left(\rho_{2}, \sigma_{v}^{2}, \sigma_{1}^{2}\right)
$$

where

$$
G_{N}=\left[\begin{array}{cccc}
g_{11}^{0} & g_{12}^{0} & g_{13}^{0} & 0 \\
g_{21}^{0} & g_{22}^{0} & g_{23}^{0} & 0 \\
g_{31}^{0} & g_{32}^{0} & g_{33}^{0} & 0 \\
g_{11}^{1} & g_{12}^{1} & 0 & g_{13}^{1} \\
g_{21}^{1} & g_{22}^{1} & 0 & g_{23}^{1} \\
g_{31}^{1} & g_{32}^{1} & 0 & g_{33}^{1}
\end{array}\right], g_{N}=\left[\begin{array}{c}
g_{1}^{0} \\
g_{2}^{0} \\
g_{3}^{0} \\
g_{1}^{1} \\
g_{2}^{1} \\
g_{3}^{1}
\end{array}\right]
$$


and

$$
\begin{aligned}
g_{11}^{i} & =\frac{2}{N(T-1)^{1-i}} \widetilde{\varepsilon}_{N} Q_{i, N} \widetilde{\bar{\varepsilon}}_{N}, g_{12}^{i}=-\frac{1}{N(T-1)^{1-i}} \widetilde{\bar{\varepsilon}}_{N} Q_{i, N} \widetilde{\bar{\varepsilon}}_{N}, \\
g_{21}^{i} & =\frac{2}{N(T-1)^{1-i}} \widetilde{\overline{\bar{\varepsilon}}}_{N} Q_{i, N} \widetilde{\bar{\varepsilon}}_{N}, g_{22}^{i}=-\frac{1}{N(T-1)^{1-i}} \widetilde{\bar{\varepsilon}}_{N} Q_{i, N} \widetilde{\overline{\bar{\varepsilon}}}_{N}, \\
g_{31}^{i} & =\frac{1}{N(T-1)^{1-i}}\left(\widetilde{\varepsilon}_{N} Q_{i, N} \widetilde{\overline{\bar{\varepsilon}}}_{N}+\widetilde{\bar{\varepsilon}}_{N} Q_{i, N} \widetilde{\bar{\varepsilon}}_{N}\right), g_{32}^{i}=-\frac{1}{N(T-1)^{1-i}} \widetilde{\bar{\varepsilon}}_{N} Q_{i, N} \widetilde{\bar{\varepsilon}}_{N}, \\
g_{13}^{i} & =1, g_{1}^{i}=\frac{1}{N(T-1)^{1-i}} \widetilde{\varepsilon}_{N} Q_{i, N} \widetilde{\varepsilon}_{N} \\
g_{23}^{i} & =\frac{1}{N} \operatorname{tr}\left(W_{N}^{\prime} W_{N}\right), g_{2}^{i}=\frac{1}{N(T-1)^{1-i}} \widetilde{\bar{\varepsilon}}_{N} Q_{i, N} \widetilde{\bar{\varepsilon}}_{N} \\
g_{33}^{i} & =0, g_{3}^{i}=\frac{1}{N(T-1)^{1-i}} \widetilde{\varepsilon}_{N} Q_{i, N} \widetilde{\bar{\varepsilon}}_{N}
\end{aligned}
$$

with

$$
\begin{aligned}
Q_{0, N} & =\left(I_{T}-\frac{J_{T}}{T}\right) \otimes I_{N} \\
Q_{1, N} & =\frac{J_{T}}{T} \otimes I_{N} .
\end{aligned}
$$

KKP specified a static model rather than a dynamic one, i.e. $\gamma=0$, and also their model does not have a spatial lag, i.e. $\rho_{1}=0$ in equation (1). In this context, under the random effects specification (3), the OLS estimator of $\beta$ is consistent. Using $\widetilde{\beta}_{O L S}$ one gets a consistent estimator of the disturbances $\widetilde{\varepsilon}=y-X \widetilde{\beta}_{O L S}$. The GM estimators of $\sigma_{1}^{2}, \sigma_{\nu}^{2}$ and $\rho_{2}$ are the solution of the sample moments (26). KKP suggest three GM estimators. The first involves only the first three moments which do not involve $\sigma_{1}^{2}$ and yield estimates of $\rho_{2}$ and $\sigma_{\nu}^{2}$. The fourth moment condition is then used to solve for $\sigma_{1}^{2}$ given estimates of $\rho_{2}$ and $\sigma_{\nu}^{2}$. The second GM estimator is based upon weighting the moment equations by the inverse of a properly normalized variance-covariance matrix of the sample moments evaluated at the true parameter values. A simple version of this weighting matrix is derived under normality of the disturbances. The third GM estimator is motivated by computational considerations and replaces a component of the weighting matrix for the second GM estimator by an identity matrix. KKP perform Monte Carlo experiments comparing ML and these three GM estimation methods. 
They find that on average, the RMSE of ML estimator and their weighted GM estimators are quite similar ${ }^{1} . \quad \xi_{N}\left(\rho_{2}, \sigma_{v}^{2}, \sigma_{1}^{2}\right)$ can be viewed as a vector of residuals. The GM estimator of $\rho_{2}$ is defined as the nonlinear least squares estimator corresponding to (26). Here, the OLS estimator of (1) is not consistent. Therefore, we modify the KKP approach as follows:

- In the first step, we use an IV or GMM estimator to get consistent estimates of $\gamma, \rho_{1}$ and $\beta$. For example, we can use the Anderson and Hsiao (1981, 1982) IV estimator by adding $W_{N} y_{t-2}$ to the list of instruments.

- In the second step, the IV or GMM residuals are used to obtain consistent estimates of the autoregressive parameter $\rho_{2}$ and the variance components $\sigma_{v}^{2}$ and $\sigma_{1}^{2}$.

- In the third step, we compute the preliminary one-step consistent Spatial GMM estimator which is given by

$$
\widehat{\delta}_{1}=\left(\Delta \tilde{X}^{\prime} Z^{*} \widehat{A}_{N} Z^{*^{\prime}} \Delta \widetilde{X}\right)^{-1} \Delta \tilde{X}^{\prime} Z^{*} \widehat{A}_{N} Z^{*^{\prime}} \Delta y
$$

where $\Delta \widetilde{X}=\left(\Delta y_{-1},\left(I_{T-2} \otimes W_{N}\right) \Delta y, \Delta x\right), \delta^{\prime}=\left(\gamma, \rho_{1}, \beta^{\prime}\right)$ and

$$
\widehat{A}_{N}=\left[Z^{* \prime}\left(I_{T-2} \otimes \widehat{H}_{N}\right)\left(G \otimes I_{N}\right)\left(I_{T-2} \otimes \widehat{H}_{N}^{\prime}\right) Z^{*}\right]^{-1}
$$

with $\widehat{H}_{N}=\widehat{B}_{N}^{-1}=\left(I_{N}-\widehat{\rho}_{2} W_{N}\right)^{-1}$.

- In the fourth step, following Arellano and Bond (1991), we replace (31) in (30) by

$$
V_{N}=\left[Z^{* \prime}\left(I_{T-2} \otimes \widehat{H}_{N}\right)(\Delta v)(\Delta v)^{\prime}\left(I_{T-2} \otimes \widehat{H}_{N}^{\prime}\right) Z^{*}\right]^{-1} .
$$

To operationalize this estimator, $\Delta v$ is replaced by differenced residuals obtained from the preliminary one-step consistent Spatial GMM estimator (30). The resulting estimator is the two-step Spatial GMM estimator

$$
\widehat{\delta}_{2}=\left(\Delta \widetilde{X}^{\prime} Z^{*} \widehat{V}_{N} Z^{*^{\prime}} \Delta \widetilde{X}\right)^{-1} \Delta \tilde{X}^{\prime} Z^{*} \widehat{V}_{N} Z^{*^{\prime}} \Delta y
$$

A consistent estimate of the asymptotic variance-covariance matrix of $\widehat{\delta}_{2}$ is given by the first term of $(32)$.

\footnotetext{
${ }^{1}$ In our Monte Carlo experiments, we report the results from the GM estimator called weighted GM estimator by Kapoor, et al. (2007) in order to save space. The differences with the other two GM estimators in our Monte Carlo experiments were minor.
} 


\section{Prediction}

For the static model with $\left(\gamma=\rho_{1}=0\right)$, Goldberger (1962) showed that, for a given $\Omega$, the best linear unbiased predictor (BLUP) for the $i$ th individual at a future period $T+\tau$ is given by:

$$
\widehat{y}_{i, T+\tau}=x_{i, T+\tau} \widehat{\beta}_{G L S}+\omega^{\prime} \Omega^{-1} \widehat{\varepsilon}_{G L S}
$$

where $\omega=E\left[\varepsilon_{i, T+\tau} \varepsilon\right]$ is the covariance between the future disturbance $\varepsilon_{i, T+\tau}$ and the sample disturbances $\varepsilon . \widehat{\beta}_{G L S}$ is the GLS estimator of $\beta$ based on $\Omega$ and $\widehat{\varepsilon}_{G L S}$ denotes the corresponding GLS residual vector. For the static RE model with $\left(\gamma=\rho_{2}=\rho_{1}=0\right)$, this predictor in (33) was considered by Wansbeek and Kapteyn (1978), Lee and Griffiths (1979) and Taub (1979). The BLUP in (33) reduces to:

$$
\widehat{y}_{i, T+\tau}=x_{i, T+\tau} \widehat{\beta}_{G L S}+\frac{\sigma_{\mu}^{2}}{\sigma_{1}^{2}}\left(\iota_{T}^{\prime} \otimes l_{i}^{\prime}\right) \widehat{\varepsilon}_{G L S}
$$

where $\sigma_{1}^{2}=T \sigma_{\mu}^{2}+\sigma_{v}^{2}$ and $l_{i}$ is the $i$ th column of $I_{N}$. The typical element of the last term of equation (34) is $\left(T \sigma_{\mu}^{2} / \sigma_{1}^{2}\right) \bar{\varepsilon}_{i, G L S}$ where $\bar{\varepsilon}_{i, G L S}=\sum_{t=1}^{T} \widehat{\varepsilon}_{t i, G L S} / T$. Therefore, the BLUP of $y_{i, T+\tau}$ for the RE model modifies the usual GLS forecasts by adding a fraction of the mean of the GLS residuals corresponding to the $i$ th individual. Baltagi, Bresson and Pirotte (2010) derived the BLUP and demonstrated that the second term of (34) is not modified if the structure of the disturbances is SAR-RE with $\left(\gamma=\rho_{1}=0\right)$. Another interesting case is when the model (1) is a spatial lag model with an error component structure (i.e. $\gamma=\rho_{2}=0$ ). The BLUP is given $b^{2}$ :

$$
\widehat{y}_{i, T+\tau}=\sum_{k=1}^{K} \widehat{\beta}_{k} \sum_{j=1}^{N} h_{i j} x_{k, j, T+\tau}+\frac{T \sigma_{\mu}^{2}}{\sigma_{1}^{2}} \sum_{j=1}^{N} h_{i j} \widehat{\varepsilon}_{j} .
$$

where $h_{i j}$ is the $(i, j)$ element of the matrix $G_{N}^{-1}=\left(I_{N}-\rho_{1} W_{N}\right)^{-1}, \sigma_{1}^{2}=$ $T \sigma_{\mu}^{2}+\sigma_{v}^{2}$ and $\widehat{\varepsilon}_{j}=(1 / T) \sum_{t=1}^{T} \widehat{\varepsilon}_{j t}$. In practice, the variance components and the spatial lag parameter $\rho_{1}$ of (35) are unknown. So, these are replaced by their ML estimates.

When $\gamma \neq 0$ (i.e. a dynamic model), and $\rho_{1} \neq 0$ (i.e. including a spatial lag on the dependent variable) and $\rho_{2} \neq 0$ (i.e. including a SAR process

\footnotetext{
${ }^{2}$ See the Appendix.
} 
on the disturbances $\varepsilon$ ), the derivation of the predictor is more complicated mainly because the lagged endogenous variable is correlated with the individual effects. Following Chamberlain (1984) and Sevestre and Trognon (1996), we derive the linear predictor of $y_{i t}$ conditional upon $\left(y_{10}, \ldots, y_{N 0}, x_{11}, \ldots, x_{N 1}, \ldots\right.$, $\left.x_{1 T}, \ldots, x_{N T}\right)$ which is given ${ }^{3}$ by

$$
\begin{aligned}
& E^{*}\left[y_{i t} \mid y_{10}, \ldots, y_{N 0}, x_{11}, \ldots, x_{N 1}, \ldots, x_{1 T}, \ldots, x_{N T}\right] \\
= & \gamma^{t} \sum_{j=1}^{N} h_{i j}^{(t)} y_{j 0}+\sum_{l=1}^{t} \gamma^{l-1} \sum_{j=1}^{N} h_{i j}^{(l)} x_{j t-l+1} \beta \\
& +\sum_{l=1}^{t} \gamma^{l-1} \sum_{j=1}^{N} p_{i j}^{(l)} E^{*}\left[\mu_{j} \mid y_{10}, \ldots, y_{N 0}\right],
\end{aligned}
$$

where $h_{i j}^{(l)}$ is the $(i, j)$ element of the matrix $\left(G_{N}^{-1}\right)^{l}, p_{i j}^{(l)}$ is the $(i, j)$ element of the matrix $\left(\left(\left(G_{N}\right)^{-1}\right)^{l} B_{N}^{-1}\right) \cdot \mu_{j}$ and $y_{j 0}$ are assumed to be uncorrelated with the sequence $\left(x_{j 1}, \ldots, x_{j T}\right), \forall j$. Following Chamberlain (1984), we assume that:

$$
E^{*}\left[\mu_{j} \mid y_{10}, \ldots, y_{N 0}\right]=\psi+\lambda_{1} y_{10}+\cdots+\lambda_{N} y_{N 0}
$$

\section{Monte Carlo Design}

In all the experiments the dependent variable $y_{i t}$ was generated from a model of the form:

$$
y_{i t}=a+\gamma y_{i t-1}+\rho_{1} \sum_{j=1}^{N} w_{i j} y_{j t}+\beta x_{i t}+\varepsilon_{i t} \quad i=1, \ldots, N ; t=1, \ldots, T,
$$

where the disturbance $\varepsilon_{i t}$ follows a SAR process:

$$
\varepsilon_{i t}=\rho_{2} \sum_{j=1}^{N} w_{i j} \varepsilon_{j t}+u_{i t}
$$

where $w_{i j}$ is the $(i, j)$ element of the spatial matrix $W_{N}$, and $u_{i t}$ has an error component structure

$$
u_{i t}=\mu_{i}+v_{i t}
$$

\footnotetext{
${ }^{3}$ To facilitate the presentation, we consider only one exogenous variable. See the Appendix.
} 
with $\mu_{i} \sim i i d . N\left(0, \sigma_{\mu}^{2}\right), v_{i t} \sim i i d . N\left(0, \sigma_{v}^{2}\right)$ and $\left(\sigma_{\mu}^{2}, \sigma_{v}^{2}\right)=(0.8,0.2),(0.2,0.8)$. The explanatory variable $x_{i t}$ is generated as:

$$
x_{i t}=\delta x_{i t-1}+\xi_{i t}
$$

with $\delta=0.6, \xi_{i t} \sim \operatorname{iid.} N\left(0, \sigma_{\xi}^{2}\right), \sigma_{\xi}^{2}=5$ and $x_{i 0}=0$. One sample size is considered $(N, T)=(100,7)$. For the coefficients of (37) and (38), we assume the values $(a, \gamma, \beta)=(1,0.2,1),(1,0.5,1), \rho_{1}=0.7,0.4,0.2$ and $\rho_{2}=0.4$. The first ten cross-sections were discarded in order to reduce the dependency on initial values. Moreover, following Kelejian and Prucha (1999), two weight matrices are used which essentially differ in their degree of sparseness. The weight matrices are labelled as " $j$ ahead and $j$ behind" with the non-zero elements being $1 / 2 j, j=1$ and 5 , hereafter respectively $W(1,1)$ and $W(5,5)$. For all experiments, 1000 replications are performed. We compute the mean, standard deviation, bias and RMSE of the coefficients $\widehat{\gamma}, \widehat{\beta}, \widehat{\rho}_{1}$ and $\widehat{\rho}_{2}$. Following Kapoor, et al. (2007), we adopt a measure of dispersion which is closely related to the standard measure of root mean square error (RMSE), but is based on quantiles. It is defined as

$$
\mathrm{RMSE}=\left[\text { bias }^{2}+\left(\frac{I Q}{1.35}\right)^{2}\right]^{1 / 2}
$$

where bias is the difference between the median and the true value of the parameter, and $I Q$ is the interquantile range defined as $c_{1}-c_{2}$ where $c_{1}$ is the 0.75 quantile and $c_{2}$ is the 0.25 quantile. Clearly, if the distribution is normal the median is the mean and, aside from a slight rounding error, $I Q / 1.35$ is the standard deviation. In this case, the measure (41) reduces to the standard RMSE.

We compare the performance of 8 estimators in our Monte Carlo experiments. These are as follows:

1. Ordinary Least Squares (OLS) which does not deal with the endogeneity of the spatial $W_{N} y$ and the endogeneity of the lagged dependent variable. OLS also ignores the individual effects and the SAR process for the disturbances.

2. The Within $(\mathrm{W})$ estimator which wipes out the individual effects, but otherwise does not deal with the endogeneity of the spatial $W_{N} y$ and the endogeneity of the lagged dependent variable nor the SAR process for the disturbances. 
3. The Arellano and Bond (1991) GMM (1) estimator which differences the individual effects, and handles the presence of the lagged dependent variable by using the orthogonality conditions (8) and (9). However, this estimator ignores the spatial $W_{N} y$ and the SAR process for the disturbances.

4. GMM (2) is an application of the Arellano and Bond (1991) estimator as in GMM (1) but including the spatial $W_{N} y$ as an extra regressor. This estimator ignores the SAR process for the disturbances.

5. GMM-SAR-RE (1) is the estimator suggested by Mutl (2006) which accounts for the lagged dependent variable and the SAR-RE process in the spirit of KKP (2007). However, this estimator ignores the spatial $W_{N} y$.

6. GMM-SAR-RE (2) is an application of the Mutl (2006) estimator as in GMM-SAR-RE (1) but including the spatial $W_{N} y$ as an extra regressor.

7. GMM-SL-RE is an estimator that uses the orthogonality conditions (8) and (9) of Arellano and Bond (1991) as well as the spatial orthogonality conditions (10) and (11). However, this estimator ignores the SARRE process for the disturbances. This GMM estimator uses a similar orthogonality conditions to that of Elhorst (2010).

8. GMM-SL-SAR-RE is an estimator that uses the orthogonality conditions (8) and (9) of Arellano and Bond (1991) as well as the spatial orthogonality conditions (10) and (11) as in GMM-SL-RE, but it also accounts for the SAR-RE structure of the disturbances using a KKP approach. This estimator is described in Section 3.

Last, we check the prediction-performance of the estimators considered. We use the RMSE criterion and compute the out of sample forecast errors for each predictor associated with the alternative estimators for one to five step ahead forecasts, see Baltagi, Bresson and Pirotte (2010). We also compute the Theil's U statistic to avoid the scaling problem of RMSE, as discussed by Trapani and Urga (2009). Average values of RMSE are calculated across $N$ for all five step forecasts. ${ }^{4}$

\footnotetext{
${ }^{4}$ Tables reporting Theil's U statistic are available upon request from the authors.
} 


\section{Monte Carlo results}

\subsection{Mean, bias and RMSE of the estimators}

Table 1 presents the mean, bias and RMSE of the coefficients $\widehat{\gamma}, \widehat{\rho}_{1}, \widehat{\rho}_{2}$ and $\widehat{\beta}$ for $(N, T)=(100,7),\left(\sigma_{\mu}^{2}, \sigma_{v}^{2}\right)=(0.8,0.2), \beta=1, \rho_{2}=0.4$ considering a $\mathrm{W}(1,1)$ matrix, one neighbor ahead and one neighbor behind.

The first panel in Table 1 shows the results for $\gamma=0.2$ (low value of the lagged dependent variable coefficient) and $\rho_{1}=0.2$ and 0.7 , (low and high values of the spatial lag coefficient). The second panel in Table 1 shows the results for $\gamma=0.5$ (high value of the lagged dependent variable coefficient). In particular, for $\rho_{1}=0.2$ in Table 1, the GMM estimator associated with model (1) (i.e. including both the spatial lag variable and SAR-RE process, namely GMM-SL-SAR-RE) has lower RMSEs for the coefficients $\rho_{1}$ and $\rho_{2}$ than those obtained for GMM-SAR-RE (2) and GMM spatial lag including RE disturbances, namely GMM-SL-RE. The RMSEs of $\gamma$ and $\beta$ are similar. The GMM-SAR-RE (2) estimator is an extension of the Mutl (2006) estimator (GMM-SAR-RE (1)) with the additional regressor $W_{N} y$. GMMSL-RE is an estimator that uses the orthogonality conditions (8) and (9) of Arellano and Bond (1991) as well as the spatial orthogonality conditions (10) and (11). However, this estimator ignores the SAR-RE process for the disturbances. Note the huge bias and RMSE for OLS, Within and GMM (1) for the coefficients $\gamma, \rho_{1}, \beta$. These results are not surprising. OLS does not deal with the endogeneity of the spatial $W_{N} y$ and the endogeneity of the lagged dependent variable. OLS also ignores the individual effects and the SAR process for the disturbances. The Within estimator does not deal with the endogeneity of the spatial lag $W_{N} y$ and the endogeneity of the temporally lagged dependent variable nor the SAR process for the disturbances. Moreover GMM (1) ignores the spatial lag $W_{N} y$ and the SAR process for the disturbances.

Consider next the outcomes in the top panel of Table 1 for $\gamma=0.2$ (i.e. low value of the lagged dependent variable coefficient) but for the high value of the spatial lag coefficient $\left(\rho_{1}=0.7\right)$. In this case, GMM-SL-SAR-RE is the best in terms of RMSE for the coefficients $\rho_{1}, \rho_{2}, \gamma$ and $\beta$. Note that the bias and RMSE for OLS, Within and GMM (1) for the coefficients $\gamma$, $\rho_{1}, \beta$ increases. It is apparent that the larger value of spatial lag coefficient improves the results produced by the GMM-SL-SAR-RE estimator.

If we consider the second panel in Table 1, i.e. $\gamma=0.5$ (high value of the 
lagged dependent variable coefficient) and $\rho_{1}=0.2$ (low value of the spatial lag coefficient), GMM-SL-SAR-RE is still the best in terms of RMSE for the coefficients $\rho_{1}, \gamma, \beta$ and $\rho_{2}$. OLS, Within and GMM (1) estimation of the coefficients $\gamma, \rho_{1}, \beta$ produces more bias and a larger RMSE compared to the outcomes with $\gamma=0.2$. If we maintain the value of $\gamma$ at 0.5 but increase $\rho_{1}$ so that it takes the value 0.4 (i.e. a higher value for the spatial lag coefficient), the GMM estimator for the model (1) (i.e. including spatial lag variable and SAR-RE process) remains the best in terms of RMSE for the coefficients $\rho_{1}$, $\gamma, \beta$ and $\rho_{2}$. In other words the results are the same, but the magnitudes are different.

Table 2 considers a lower level of individual heterogeneity, $\left(\sigma_{\mu}^{2}, \sigma_{v}^{2}\right)=$ $(0.2,0.8)$ but otherwise leaves all the other parameters as in Table 1 . In terms of RMSE, the GMM-SL-SAR-RE estimator remains the best whatever the values of $\rho_{1}$ and $\gamma$. Overall, the RMSEs are greater than those of Table 1 , where we assumed that $\left(\sigma_{\mu}^{2}, \sigma_{v}^{2}\right)=(0.8,0.2)$.

\subsection{Forecast accuracy}

Table 3 gives the RMSEs for the one year and five year ahead forecasts along with the average RMSE for all 5 years. These are out-of-sample forecasts when the true model is (1) (i.e. including both temporally and spatially lagged dependent variables) with SAR-RE disturbances. The sample size is $N=100$ and $T=7$, the weights matrix is $W(1,1)$, i.e., one neighbor behind and one neighbor ahead.

If we consider the case $\left(\sigma_{\mu}^{2}, \sigma_{v}^{2}\right)=(0.8,0.2)$ and $\left(\gamma, \rho_{1}\right)=(0.2,0.2)$, the lowest RMSE is that of GMM-SL-SAR-RE, followed closely by the RSMEs produced by the GMM-SAR-RE and GMM-SL-RE estimators. It appears that misspecifying the disturbances affects the forecast performance, since the RMSEs of the OLS and Within estimators are approximately double those produced by GMM-SL-SAR-RE. If the spatial lag parameter $\rho_{1}$ increases from 0.2 to 0.7 , the RMSEs increase sharply, but the ranking remains intact. If $\left(\gamma, \rho_{1}\right)=(0.5,0.2)$ or $\left(\gamma, \rho_{1}\right)=(0.5,0.4)$, the results remain essentially the same, although the magnitudes are different. The GMM estimators that take endogeneity and/or heterogeneity into account perfom better than the OLS and Within estimators that do not. This forecast comparison is robust to whether we are predicting one period ahead or five periods ahead and is also reflected in the average over the five years. Thus the gain in forecast performance is substantial once we account for endogeneity and het- 
erogeneity.

If we consider the case a low level of individual heterogeneity, i.e. $\left(\sigma_{\mu}^{2}, \sigma_{v}^{2}\right)$ $=(0.2,0.8)$, the lowest RMSE remains that of GMM-SL-SAR-RE. However, the difference between the GMM-SL-SAR-RE and GMM-SL-RE outcomes is reduced.

\subsection{Sensitivity Analysis}

For the various estimators considered, Tables 4 and 5 report the mean, bias and RMSE results, as was done for Tables 1 and 2 except that the weight matrix is changed from a $W(1,1)$ to $W(5,5)$, i.e., five neighbors behind and five neighbors ahead. Table 6 reports the forecast RMSE results in the same way as those of Table 3 except that again the weight matrix is $W(5,5)$ rather than $W(1,1)$. To summarize, we find that the results are essentially the same, although the magnitudes are different.

\section{Empirical illustration}

\subsection{The Model}

Our empirical illustration is motivated by recent work seeking to apply, and test the viability of, contemporary economic geography theory, as presented in the seminal work of Fujita, Krugman and Venables (1999). This is commonly referred to as the new economic geography (NEG). We draw on one of the model specifications of Fingleton and Fischer (2010), although we do not make a formal link to the NEG theory in this paper. More formally we estimate the following dynamic spatial panel model:

$$
\begin{aligned}
\ln y_{i t}= & a+\gamma \ln y_{i t-1}+\rho_{1} \sum_{j=1}^{N} w_{i j} \ln y_{j t}+\beta_{1} \ln P_{i t}+\beta_{2} \ln S_{i t} \\
& +\beta_{3} \ln n_{i t}+\beta_{4} \ln s_{i t}+\varepsilon_{i t} \quad i=1, \ldots, N ; t=1, \ldots, T
\end{aligned}
$$

where $y_{i t}$ is Gross Value Added (GVA) per worker for the ith region $(i=$ $1, \ldots, N)$ and t-th time period $(t=1, \ldots, T) . P_{i t}$ is market potential ${ }^{5}, S_{i t}$ is schooling, $n_{i t}$ is the (adjusted) population growth rate, and $s_{i t}$ is the investment rate. Moreover, following Koch (2008), who extended the neoclassical

\footnotetext{
${ }^{5}$ The measurement of market potential is difficult and complex. For simplicity, we use an earlier definition of market potential initially attributed to Harris (1954), which is
} 
growth model to pick up spatial spillover effects, we also include the spatial lag of the dependent variable, given by the matrix product of the $W_{N}$ matrix and $y_{i t} . W_{N}$ is a first order contiguity matrix, which considers that two geographic regions $i$ and $j$ are neighbors if they directly share a border. More precisely, the weights matrix is binary, with $w_{i j}=1$ when $i$ and $j$ are neighbors and $w_{i j}=0$ when they are not. By convention, diagonal elements are null: $w_{i i}=0$ and the weights are normalized such that the elements of each row sum to 1 (i.e. row-normalized). As in Fingleton and Fischer (2010), our panel specification also includes a spatial SAR error process with

$$
\varepsilon_{i t}=\rho_{2} \sum_{j=1}^{N} w_{i j} \varepsilon_{j t}+u_{i t}
$$

and

$$
u_{i t}=\mu_{i}+v_{i t}
$$

in which the remainder term $u_{i t}$ is composed of a region-specific individual effect $\mu_{i}$ and an idiosyncratic random shock $v_{i t}$.

\subsection{Data Description}

The data, which originate from Eurostat's Region database, Statistics Norway and the Swiss Office Féderal de la Statistique, comprise 255 NUTS-2 regions, observed over the period 1995-2003, and covering 25 European countries, hence $N=255$ and $T=9$. The short time dimension is a consequence of the lack of reliable data for Central and Eastern Europe regions, and also because of the fundamental reorganization of some formerly centrally planned command economies. The data cover Austria (nine regions), Belgium (11 regions), Czech Republic (eight regions), Denmark (one region), Estonia (one region), Finland (five regions), France (22 regions), Germany (40 regions), Greece (13 regions), Hungary (seven regions), Ireland (two regions), Italy (20

regularly used in the applied literature:

$$
P_{i t}=\sum_{i \neq j}^{N} G_{i t} d_{i j}^{-\tau}
$$

in which $G_{i t}$ is the "size" of the economy in region $i$, and $d_{i j}$ is the "distance" between region $i$ and region $j$. 
regions), Latvia (one region), Lithuania (one region), Luxembourg (one region), Netherlands (12 regions), Norway (seven regions), Poland (16 regions), Portugal (five regions), Slovakia (four regions), Slovenia (one region), Spain (16 regions), Sweden (eight regions), Switzerland (seven regions), and UK (37 regions). ${ }^{6}$

The dependent variable $y_{i t}$ is measured by Gross Value Added (GVA) per worker. GVA is the net result of output at basic prices minus intermediate consumption at purchasers' prices, following the approach used in the European System of Accounts [ESA] 1995. The adjusted population growth rate $n_{i t}$ is based on the rate of growth of the working-age population in region $i$ at time $t$, with working age defined as 15 to 64 years. For details, see Fingleton and Fischer (2010). The investment rate $s_{i t}$ is the share of gross investment in gross regional product. We proxy labour efficiency and workforce skill by the level of educational attainment variable $S_{i t}$, which is defined as the share of population (15 years and older) with higher education as given by the ISCED 1997 classes 5 and 6.

Market potential is a product of the total regional GVA, $G_{i t}$, and $d_{i j}^{-\tau}$ in which $d_{i j}$ is the great-circle distance from (the economic centre of) region $i$ to (the economic centre of) region $j$. For region $i$, we sum across all other regions, excluding region $i$, so as to maximize exogeneity of $P_{i t}$ with respect to $y_{i t}$. We assume that the rate of discount with distance $(\tau=0.5)$. We experimented with alternative values of $\tau$ and they gave similar outcomes to those described below. ${ }^{7}$

\subsection{Results}

We estimate our model (42) using the period 1995-2001, leaving out the last two years for the purpose of out-of-sample forecasting. Given the dynamic specification, one's first instinct could be to apply the well-known Arellano and Bond (1991) estimator. However, as we have already shown in our Monte Carlo study, the GMM (2) estimator does not produce satisfactory estimates. This is confirmed again in the empirical example in Table 7 . Note that out of the four estimators summarised in Table 7, two ignore the spatial error process (i.e. GMM (2) and GMM-SL-RE), and two take the error process into

\footnotetext{
${ }^{6}$ We are grateful to Professor Manfred Fischer for his help in producing these data.

${ }^{7}$ Note that our model differs from that of Fingleton and Fischer (2010) in two respects. They estimate a static model whereas our model is dynamic. They use a different NEG market potential than our Harris measure.
} 
account (i.e. GMM-SAR-RE (2) and GMM-SL-SAR-RE). The estimated $\rho_{2}$ is equal to 0.877 and 0.714 respectively for GMM-SAR-RE (2) and GMMSL-SAR-RE. Also GMM (2) relies only on the orthogonality conditions (8) and (9), whereas GMM-SL-RE and GMM-SL-SAR-RE employ the additional spatial orthogonality conditions (10) and (11). Table 7 clearly shows considerable differences among the estimators. In particular, the temporal lag coefficient estimates of $\gamma$ are smaller for the estimators that exclude the SAR disturbance process. In contrast, the spatial lag $\left(\rho_{1}\right)$ estimates are larger for estimators that exclude the SAR disturbance process. The larger estimated $\rho_{1}$ produced under GMM (2) and GMM-SL-RE suggest that the spatial lag is capturing spatial effects that would otherwise be partially captured by an error process model. Note that while the point estimates differ, both temporal and spatial lags are statistically significant for all four estimators. Note also that the Harris-market potential variable is only significant for GMMSL-SAR-RE. Moreover, the stationarity conditions are only satisfied for this estimator. This is in line with what the NEG theory suggests, and ties in with the interpretation provided by the static panel estimates of Fingleton and Fischer (2010). The educational attainment variable $S_{i t}$ is significant for GMM-SAR-RE (2) and GMM-SL-RE. As the schooling variable only changes slowly through time, it appears that the anticipated positive impact of schooling may not be particularly well identified. Of the other variables, population growth $n_{i t}$ and the investment rate $s_{i t}$, these represent the rival non-nested neoclassical growth model, so we do not anticipate significant parameter estimates, but expect the neoclassical model to be encompassed by the dominant rival, as found in the formal NEG context by Fingleton and Fischer (2010). In the current dynamic setting, it turns out that while adjusted population growth is insignificant, so that we cannot reject the null hypothesis that $\beta_{3}=0$, on the whole and contrary to expectation the investment rate is a significant variable, with $\beta_{4}>0$. On reflection this is perhaps not surprising given the absence of capital investment in basic NEG theory.

The superiority of our GMM-SL-SAR-RE estimator, which has already been highlighted in our Monte Carlo analysis, is also apparent for our empirical example. This is illustrated using the RMSE for the out-of-sample forecast period. This RMSE is calculated for each of the 255 NUTS-2 regions for each of the two years. The last three lines of Table 7 give the across-region RMSE means for each year, and the two-year average. From this it is clear that, when one compares model predictions and the actual GVA per worker outcomes, the GMM-SL-SAR-RE produces by far the best 
RMSE forecasts.

\section{Conclusion}

Our Monte Carlo study finds that when the true model is a dynamic first order spatial autoregressive specification with SAR-RE disturbances, estimators that ignore the endogeneity of the spatial lag $W_{N} y$ and the endogeneity of the temporally lagged dependent variable perform badly in terms of bias and RMSE. For our experiments, accounting for heterogeneity and endogeneity improve the forecast performance by a big margin; accounting only for spatial correlation in the disturbances also improves the forecast, but by a smaller margin. Recognising the presence of a spatially lagged dependent variable among the regressors has an important effect. Ignoring both sources of spatial dependence significantly worsens the forecasting performance and leads to a huge bias in the estimated coefficients. So, a misspecified estimator, especially in terms of spatial effects, has severe consequences in terms of estimation and forecasting for the applied economist. We come to the conclusion, supported by our empirical example, that our dynamic spatial GMM estimator performs well and is recommended in practice. 


\section{References}

[1] Anderson, T.W. and C. Hsiao, 1981, Estimation of dynamic models with error components, Journal of the American Statistical Association 76, 598-606.

[2] Anderson, T.W. and C. Hsiao, 1982, Formulation and estimation of dynamic models using panel data, Journal of Econometrics 18, 47-82.

[3] Anselin, L., 1988, Spatial Econometrics: Methods and Models, Kluwer Academic Publishers, Dordrecht.

[4] Anselin, L., J. Le Gallo and H. Jayet, 2008, Spatial panel econometrics. Chapter 19 in L. Mátyás and P. Sevestre, eds., The Econometrics of Panel Data: Fundamentals and Recent Developments in Theory and Practice, Springer-Verlag, Berlin, 625-660.

[5] Arellano, M. and S. Bond, 1991, Some tests of specification for panel data: Monte Carlo evidence and an application to employment, Review of Economic Studies 58, 277-297.

[6] Arellano, M. and B. Honoré, 2001, Panel data models: some recent developments, in Handbook of Econometrics, J.J. Heckman and E.E. Leamer eds., Vol. 5, North-Holland, Amsterdam, 3229-3296.

[7] Baltagi, B.H., 2008, Econometric Analysis of Panel Data, Wiley and Sons, Chichester.

[8] Baltagi B.H. and A. Pirotte, 2010, Panel data inference under spatial dependence, Economic Modelling 27, 1368-1381.

[9] Baltagi B.H., G. Bresson and A. Pirotte, 2010, Forecasting with spatial panel data, Computational Statistics and Data Analysis, in press, available online 30 August 2010.

[10] Baltagi, B.H. and D. Li, 2006, Prediction in the panel data model with spatial correlation: The case of liquor, Spatial Economic Analysis 1, 175-185.

[11] Blundell, R. and S. Bond, 1998, Initial conditions and moment restrictions in dynamic panel data models, Journal of Econometrics 87, 115143. 
[12] Blundell, R., S. Bond and F. Windmeijer, 2000, Estimation in dynamic panel data models: improving on the performance of the standard GMM estimator, in Advances in Econometrics: Nonstationary Panels, Panel Cointegration and Dynamic Panels, B.H. Baltagi ed., Vol. 15, JAI Elsevier, Amsterdam, 8-92.

[13] Chamberlain, G., (1984), Panel data, in The Handbook of Econometrics, Z. Griliches and M. Intriligator, eds., Chapter 22, North-Holland, Amsterdam, 1247-1318.

[14] Elhorst, J.P., 2005, Unconditional maximum likelihood estimation of linear and log-linear dynamic models for spatial models, Geographical Analysis 37, 85-106.

[15] Elhorst, J.P., 2010, Dynamic panels with endogenous interaction effects when $T$ is small, Regional Science and Urban Economics 40, 272-282.

[16] Fingleton, B., 2009, Prediction using panel data regression with spatial random effects, International Regional Science Review 32, 195-220.

[17] Fingleton, B., 2010, Predicting the geography of house prices, SERC Discussion paper $n^{\circ} 45$, The London School of Economics and Political Science.

[18] Fingleton, B. and M. Fischer, 2010, Neoclassical theory versus new economic geography. Competing explanations of cross-regional variation in economic development, Annals of Regional Science 44, 467-491.

[19] Fujita, M., P.R. Krugman and A. Venables, 1999, In The Spatial Economy: Cities, Regions, and International Trade, Cambridge Massachusetts: MIT press.

[20] Goldberger, A.S., 1962, Best linear unbiased prediction in the generalized linear regression model, Journal of the American Statistical Association 57, 369-375.

[21] Harris, C., 1954, The market as a factor in the localization of industry in the United States, Annals of the Association of American Geographers 64, 315-348. 
[22] Harris, M.N., P. Sevestre and L. Mátyás, 2008, Dynamic models for short panels. Chapter 7 in L. Mátyás and P. Sevestre, eds., The Econometrics of Panel Data: Fundamentals and Recent Developments in Theory and Practice, Springer-Verlag, Berlin, 249-278.

[23] Hsiao, C., 2003, Analysis of Panel Data, Cambridge University Press, Cambridge.

[24] Jacobs, J.P.A.M., J.E. Ligthart and H. Vrijburg, 2009, Dynamic panel data models featuring endogenous interaction and spatially correlated errors, Working Paper n09-15, International Studies Program, Georgia State University.

[25] Kapoor, M., H.H. Kelejian and I.R. Prucha, 2007, Panel data models with spatially correlated error components, Journal of Econometrics 140, $97-130$.

[26] Kelejian, H.H. and I.R. Prucha, 1999, A generalized moments estimator for the autoregressive parameter in a spatial model, International Economic Review 40, 509-533.

[27] Kholodilin, K.A., B. Siliverstovs and S. Kooths, 2008, A dynamic panel data approach to the forecasting of the DGP of German Länder, Spatial Economic Analysis 3, 195-207.

[28] Koch, W., 2008, Development accounting with spatial effects, Spatial Economic Analysis 3, 321-341.

[29] Kukenova, M. and J.-A. Monteiro, 2009, Spatial dynamic panel model and system GMM: a Monte Carlo investigation, Munich Personal RePEc Archive paper $\mathrm{n}^{\circ} 14319$. http://mpra.ub.uni-muenchen.de/14319/.

[30] Lee, L.-F. and W.E. Griffiths, 1979, The prior likelihood and best linear unbiased prediction in stochastic coefficient linear models, working paper, Department of Economics, University of Minnesota.

[31] Lee, L.-F. and J. Yu, 2010a, Estimation of spatial autoregressive panel data models with fixed effects, Journal of Econometrics 154, 165-185.

[32] Lee, L.-F. and J. Yu, 2010b, Some recent developments in spatial panel data models, Regional Science and Urban Economics 5, 255-271. 
[33] LeSage, J.P. and R.K. Pace, 2009, Introduction to Spatial Econometrics, Chapman \& Hall/CRC Press, Boca Raton.

[34] Longhi S. and P. Nijkamp, 2007, Forecasting regional labor market developments under spatial autocorrelation, International Regional Science Review 30, 100-119.

[35] Mutl, J., 2006, Dynamic panel data models with spatially correlated disturbances, $\mathrm{PhD}$, University of Maryland.

[36] Nickell, S., 1981, Biases in dynamic model with fixed effects, Econometrica 49, 1417-1426.

[37] Sevestre, P. and A. Trognon, 1983, Propriétés de grands échantillons d'une classe d'estimateurs des modèles autorégressifs à erreurs composées, Annales de l'INSEE 50, 25-48.

[38] Sevestre, P. and A. Trognon, 1985, A note on autoregressive error component models, Journal of Econometrics 28, 231-245.

[39] Sevestre, P. and A. Trognon, 1996, Dynamic linear models, in The Econometrics of Panel Data: a Handbook of Theory with Applications, L. Màtyàs and P. Sevestre, eds., Chapter 7, Kluwer Academic Publishers, Dordrecht, 121-144.

[40] Taub, A.J., 1979, Prediction in the context of the variance-components model, Journal of Econometrics 10, 103-108.

[41] Trapani, L. and G. Urga, 2009, Optimal forecasting with heterogeneous panels: a Monte Carlo study, International Journal of Forecasting 25, 567-586.

[42] Trognon, A., 1978, Miscellaneous asymptotic properties of ordinary least squares and maximum likelihood estimators in dynamic error components models, Annales de l'INSEE 30-31, 631-657.

[43] Védrine, L. and S. Bouayad-Agha, 2010, Estimation strategies for a spatial dynamic panel data using GMM. A new approach to the convergence issue of European regions, Spatial Economic Analysis, 5, 205-227. 
[44] Wansbeek, T.J. and A. Kapteyn, 1978, The seperation of individual variation and systematic change in the analysis of panel data, Annales de l'INSEE 30-31, 659-680.

[45] Yu, J., R. de Jong and L. Lee, 2008, Quasi-Maximum Likelihood Estimators for spatial dynamic panel data with fixed effects when both $N$ and $T$ large, Journal of Econometrics 146, 118-134. 


\section{Appendix}

In matrix form, we can write (1), (2) and (3) as

$$
y=\gamma y_{-1}+\rho_{1}\left(I_{T-1} \otimes W_{N}\right) y+X \beta+\varepsilon
$$

with

$$
\varepsilon=\rho_{2}\left(I_{T-1} \otimes W_{N}\right) \varepsilon+u
$$

or

$$
\varepsilon=\left(I_{T-1} \otimes B_{N}^{-1}\right) u
$$

with $B_{N}=\left(I_{T-1}-\rho_{2} W_{N}\right)$, and

$$
u=\left(\iota_{T-1} \otimes I_{N}\right) \mu+v
$$

where $y=\left(y_{2}^{\prime}, \ldots, y_{T}^{\prime}\right)^{\prime}, y_{-1}=\left(y_{1}^{\prime}, \ldots, y_{T-1}^{\prime}\right)^{\prime}, X=\left(X_{2}^{\prime}, \ldots, X_{T}^{\prime}\right)^{\prime}, \mu=$ $\left(\mu_{1}, \ldots, \mu_{N}\right)^{\prime}, v=\left(v_{2}^{\prime}, \ldots, v_{T}^{\prime}\right)^{\prime}, \iota_{T-1}$, a $((T-1) \times 1)$ vector of ones. The variance-covariance matrix of $(49)$ is given by:

$$
\Omega_{u}=\sigma_{\mu}^{2}\left(J_{T-1} \otimes I_{N}\right)+\sigma_{v}^{2} I_{(T-1) N}=\sigma_{v}^{2} Q_{0, N}+\sigma_{1}^{2} Q_{1, N}
$$

where

$$
\begin{aligned}
Q_{0, N} & =\left(I_{T-1}-\frac{J_{T-1}}{T-1}\right) \otimes I_{N}, Q_{1, N}=\frac{J_{T-1}}{T-1} \otimes I_{N}, \\
\sigma_{1}^{2} & =(T-1) \sigma_{\mu}^{2}+\sigma_{v}^{2} .
\end{aligned}
$$

Following the properties of the operators $Q_{0, N}$ and $Q_{1, N}$, we can write:

$$
\Omega_{u}^{-1}=\sigma_{v}^{-2} Q_{0, N}+\sigma_{1}^{-2} Q_{1, N}
$$

and

$$
\Omega_{u}^{-1 / 2}=\sigma_{v}^{-1} Q_{0, N}+\sigma_{1}^{-1} Q_{1, N}
$$

or

$$
\sigma_{v} \Omega_{u}^{-1 / 2}=I_{(T-1) N}-\theta Q_{1, N}
$$

where $\theta=1-\sigma_{v} / \sigma_{1}$, see Baltagi (2008). 
- For the static case $\rho_{1}=\gamma=0$, Baltagi, Bresson and Pirotte (2010) derived the Best Linear Unbiased Predictor (BLUP). More precisely, this predictor is given by:

$$
\widehat{y}_{i, T+\tau}=x_{i, T+\tau} \widehat{\beta}_{G L S}+\frac{\sigma_{\mu}^{2}}{\sigma_{1}^{2}} b_{i}\left(\iota_{T}^{\prime} \otimes B_{N}\right) \widehat{\varepsilon}_{G L S}
$$

where $b_{i}$ is the $i$ th row of the matrix $B_{N}^{-1}=\left(I_{N}-\rho_{2} W_{N}\right)^{-1}$. They showed that the second term of (56) has the same form as that of the RE model whatever the structure of the disturbances (i.e. SAR-RE, $\rho_{2} \neq 0$, or SMA-RE, $\lambda \neq 0$ ). Thus, for a cross-section, the second term of (56) in matrix form is:

$$
\frac{\sigma_{\mu}^{2}}{\sigma_{1}^{2}} B_{N}^{-1}\left(\iota_{T}^{\prime} \otimes B_{N}\right) \widehat{\varepsilon}_{G L S}=\frac{\sigma_{\mu}^{2}}{\sigma_{1}^{2}}\left(\iota_{T}^{\prime} \otimes I_{N}\right) \widehat{\varepsilon}_{G L S} .
$$

- When $\gamma=0$, the model in (46) reduces to the spatial lag model with SAR disturbances. Thus, the term in (57) reduces to:

$$
\begin{aligned}
\frac{\sigma_{\mu}^{2}}{\sigma_{1}^{2}} G_{N}^{-1}\left(\iota_{T}^{\prime} \otimes I_{N}\right) \widehat{\varepsilon} & =\frac{\sigma_{\mu}^{2}}{\sigma_{1}^{2}}\left(\iota_{T}^{\prime} \otimes G_{N}^{-1}\right) \widehat{\varepsilon} \\
& =\frac{T \sigma_{\mu}^{2}}{\sigma_{1}^{2}}\left(\begin{array}{c}
\sum_{j=1}^{N} h_{1 j} \widehat{\varepsilon}_{j .} \\
\sum_{j=1}^{N} h_{2 j} \widehat{\varepsilon}_{j .} \\
\vdots \\
\sum_{j=1}^{N} h_{N j} \widehat{\varepsilon}_{j .}
\end{array}\right)
\end{aligned}
$$

where $\widehat{\varepsilon}_{j .}=(1 / T) \sum_{t=1}^{T} \widehat{\varepsilon}_{j t}, h_{i j}$ is the $(i, j)$ element of the matrix $G_{N}^{-1}=$ $\left(I_{N}-\rho_{1} W_{N}\right)^{-1}$ and $\sigma_{1}^{2}=T \sigma_{\mu}^{2}+\sigma_{v}^{2}$. Using (56) and (59), the Best Linear Unbiased Predictor for $y_{T+\tau}$ is given by:

$$
y_{T+\tau}=G_{N}^{-1}\left[X_{T+\tau} \widehat{\beta}+\frac{\sigma_{\mu}^{2}}{\sigma_{1}^{2}}\left(\iota_{T}^{\prime} \otimes I_{N}\right) \widehat{\varepsilon}\right] .
$$

This can be written for region $i$ at time $T+\tau$ as follows:

$$
\widehat{y}_{i, T+\tau}=\sum_{k=1}^{K} \widehat{\beta}_{k} \sum_{j=1}^{N} h_{i j} x_{k, j, T+\tau}+\frac{T \sigma_{\mu}^{2}}{\sigma_{1}^{2}} \sum_{j=1}^{N} h_{i j} \widehat{\varepsilon}_{j .} .
$$

In practice, the variance components and the spatial lag parameter $\rho_{1}$ are unknown. So, these are replaced by their estimated values. 
- When $\gamma \neq 0$ (i.e. a dynamic model) and $\rho_{1}=\rho_{2}=0$ (i.e. no spatial lag on dependent variable and no SAR process on the disturbances $\varepsilon$ ), the derivation of the predictor is more complicated, see Sevestre and Trognon $(1996)^{8}$. The lagged endogenous variable is correlated with the individual effects. In this case, the autoregressive error components model $^{9}$ can be rewritten as follows:

$$
y_{i t}=\gamma^{t} y_{i 0}+\beta \sum_{l=0}^{t-1} \gamma^{l} x_{i t-l}+\frac{1-\gamma^{t}}{1-\gamma} \mu_{i}+\sum_{l=0}^{t-1} \gamma^{l} v_{i t-l}
$$

Sevestre and Trognon (1996) give the linear predictor of $y_{i t}$ conditional upon $y_{i 0}, x_{i 1}, \ldots, x_{i T}$. This predictor is given by:

$$
\begin{aligned}
E^{*}\left[y_{i t} \mid y_{i 0}, x_{i 1}, \ldots, x_{i T}\right]= & \gamma^{t} y_{i 0}+\beta \sum_{l=0}^{t-1} \gamma^{l} x_{i t-l} \\
& +\frac{1-\gamma^{t}}{1-\gamma} \frac{E\left[\mu_{i} y_{i 0}\right]}{V\left[y_{i 0}\right]}\left(y_{i 0}-E\left[y_{i 0}\right]\right),
\end{aligned}
$$

if the individual effect $\mu_{i}$ is uncorrelated with the sequence $\left(x_{i 1}, \ldots, x_{i T}\right)$ and $y_{i 0}$ is predetermined. The last term of equation (63) is obtained using:

$$
E^{*}\left[v_{i t-l} \mid y_{i 0}, x_{i 1}, \ldots, x_{i T}\right]=0, l=0, \ldots, t-1
$$

and

$$
E^{*}\left[\mu_{i} \mid y_{i 0}, x_{i 1}, \ldots, x_{i T}\right]=E^{*}\left[\mu_{i} \mid y_{i 0}\right] .
$$

Following Chamberlain (1984, p. 1255), if we consider the least squares regression of $\mu_{i}$ on $y_{i 0}$, the population counterpart is:

$$
E^{*}\left[\mu_{i} \mid y_{i 0}\right]=\pi_{0}+\pi_{1} y_{i 0}
$$

where

$$
\pi_{1}=\frac{E\left[\mu_{i} y_{i 0}\right]}{V\left[y_{i 0}\right]}
$$

and

$$
\pi_{0}=E\left[\mu_{i}\right]-\pi_{1} E\left[y_{i 0}\right]=-\pi_{1} E\left[y_{i 0}\right] \text { since } E\left[\mu_{i}\right]=0, \forall i .
$$

\footnotetext{
${ }^{8}$ See also Harris, Màtyàs and Sevestre (2008).

${ }^{9}$ To facilitate the presentation, they considered only one exogenous variable.
} 
Substituting (67) and (68) in (66), we obtain:

$$
\begin{aligned}
E^{*}\left[\mu_{i} \mid y_{i 0}\right] & =\pi_{0}+\pi_{1} y_{i 0} \\
& =-\pi_{1} E\left[y_{i 0}\right]+\pi_{1} y_{i 0} \\
& =\pi_{1}\left(y_{i 0}-E\left[y_{i 0}\right]\right) \\
& =\frac{E\left[\mu_{i} y_{i 0}\right]}{V\left[y_{i 0}\right]}\left(y_{i 0}-E\left[y_{i 0}\right]\right) .
\end{aligned}
$$

Under stationarity assumptions, see Sevestre and Trognon (1983, p. $34)$, we can use:

$$
\begin{aligned}
y_{i 0} & =\frac{\mu_{i}}{1-\gamma}+\frac{v_{i 0}}{\sqrt{1-\gamma^{2}}} \\
E\left[y_{i 0}^{2}\right] & =\frac{\sigma_{\mu}^{2}}{(1-\gamma)^{2}}+\frac{\sigma_{v}^{2}}{1-\gamma^{2}} \\
E\left[\mu_{i} y_{i 0}\right] & =\frac{\sigma_{\mu}^{2}}{1-\gamma} .
\end{aligned}
$$

- When $\gamma \neq 0, \rho_{1} \neq 0$ and $\rho_{2} \neq 0$, equation (46) can be also written as

$$
\begin{aligned}
y-\rho_{1}\left(I_{T-1} \otimes W_{N}\right) y & =\gamma y_{-1}+X \beta+\varepsilon \\
y & =\left[I_{T-1} \otimes G_{N}^{-1}\right]\left[\gamma y_{-1}+X \beta+\varepsilon\right]
\end{aligned}
$$

and $G_{N}=\left(I_{N}-\rho_{1} W_{N}\right)$, or

$$
y=\gamma y_{-1}^{*}+X^{*} \beta+\varepsilon^{*}
$$

where

$$
\begin{aligned}
y_{-1}^{*} & =\left[I_{T-1} \otimes G_{N}^{-1}\right] y_{-1} \\
X^{*} & =\left[I_{T-1} \otimes G_{N}^{-1}\right] X \\
\varepsilon^{*} & =\left[I_{T-1} \otimes G_{N}^{-1}\right] \varepsilon
\end{aligned}
$$

or

$$
\varepsilon^{*}=\left[I_{T-1} \otimes\left(B_{N} G_{N}\right)^{-1}\right] u .
$$

The recurrent equation (74) leads for a cross-section $t$ to:

$$
\begin{aligned}
y_{t}= & \gamma^{t} A^{t} y_{0}+\gamma^{t-1} A^{t} X_{1} \beta+\cdots+\gamma^{0} A X_{t} \beta \\
& +\gamma^{t-1} A^{t} \varepsilon_{1}+\cdots+\gamma^{0} A \varepsilon_{t} \\
= & \gamma^{t} A^{t} y_{0}+\sum_{l=1}^{t} \gamma^{l-1} A^{l} X_{t-l+1} \beta+\sum_{l=1}^{t} \gamma^{l-1} A^{l} \varepsilon_{t-l+1}
\end{aligned}
$$


where $y_{0}$ is an $N \times 1$ vector of initial observations and $A=G_{N}^{-1}$. If we consider only one exogenous variable, equation (79) gives for an individual $i$ at time $t$ :

$$
y_{i t}=\gamma^{t} \sum_{j=1}^{N} h_{i j}^{(t)} y_{j 0}+\sum_{l=1}^{t} \gamma^{l-1} \sum_{j=1}^{N} h_{i j}^{(l)} x_{j t-l+1} \beta+\sum_{l=1}^{t} \gamma^{l-1} \sum_{j=1}^{N} h_{i j}^{(l)} \varepsilon_{j t-l+1}
$$

where $h_{i j}^{(l)}$ is the $(i, j)$ element of the matrix $\left(G_{N}^{-1}\right)^{l}$. If we consider the SAR-RE structure of the disturbances $\varepsilon$ (i.e. (78) and (49)), the last term of (80) can be rewritten as

$$
\sum_{l=1}^{t} \gamma^{l-1} \sum_{j=1}^{N} h_{i j}^{(l)} \varepsilon_{j t-l+1}=\sum_{l=1}^{t} \gamma^{l-1} \sum_{j=1}^{N} p_{i j}^{(l)} \mu_{j}+\sum_{l=1}^{t} \gamma^{l-1} \sum_{j=1}^{N} p_{i j}^{(l)} v_{j t-l+1}
$$

with $p_{i j}^{(l)}$ is the $(i, j)$ element of the matrix $\left(\left(\left(G_{N}\right)^{-1}\right)^{l} B_{N}^{-1}\right)$. The linear predictor of $y_{i t}$ conditional upon $\left(y_{10}, \ldots, y_{N 0}, x_{11}, \ldots, x_{N 1}, \ldots, x_{1 T}, \ldots, x_{N T}\right)$ is given by

$$
\begin{aligned}
& E^{*}\left[y_{i t} \mid y_{10}, \ldots, y_{N 0}, x_{11}, \ldots, x_{N 1}, \ldots, x_{1 T}, \ldots, x_{N T}\right] \\
= & \gamma^{t} \sum_{j=1}^{N} h_{i j}^{(t)} y_{j 0}+\sum_{l=1}^{t} \gamma^{l-1} \sum_{j=1}^{N} h_{i j}^{(l)} x_{j t-l+1} \beta \\
& +E^{*}\left[\varepsilon_{i t}^{*} \mid y_{10}, \ldots, y_{N 0}, x_{11}, \ldots, x_{N 1}, \ldots, x_{1 T}, \ldots, x_{N T}\right],
\end{aligned}
$$

$\mu_{j}$ and $y_{j 0}$ are assumed to be uncorrelated with the sequence $\left(x_{j 1}, \ldots, x_{j T}\right)$, $\forall j$. If we use similar assumptions to those of (64) and (65), we obtain:

$$
\begin{aligned}
& E^{*}\left[y_{i t} \mid y_{10}, \ldots, y_{N 0}, x_{11}, \ldots, x_{N 1}, \ldots, x_{1 T}, \ldots, x_{N T}\right] \\
= & \gamma^{t} \sum_{j=1}^{N} h_{i j}^{(t)} y_{j 0}+\sum_{l=1}^{t} \gamma^{l-1} \sum_{j=1}^{N} h_{i j}^{(l)} x_{j t-l+1} \beta \\
& +\sum_{l=1}^{t} \gamma^{l-1} \sum_{j=1}^{N} p_{i j}^{(l)} E^{*}\left[\mu_{j} \mid y_{10}, \ldots, y_{N 0}\right] .
\end{aligned}
$$

Following Chamberlain (1984, p. 1257), we assume that:

$$
\begin{aligned}
E^{*}\left[\mu_{j} \mid y_{10}, \ldots, y_{N 0}\right] & =\psi+\lambda_{1} y_{10}+\cdots+\lambda_{N} y_{N 0} \\
& =\psi+\lambda^{\prime} y_{0}
\end{aligned}
$$


where $\lambda$ is an $N \times 1$ vector with

$$
\lambda=V\left[y_{0}\right]^{-1} \operatorname{cov}\left[\mu_{j}, y_{0}\right]=V\left[y_{0}\right]^{-1} E\left[\mu_{j} y_{0}\right]
$$

and

$$
\psi=E\left[\mu_{j}\right]-\lambda_{1} E\left[y_{10}\right]-\cdots-\lambda_{N} E\left[y_{N 0}\right]
$$

Following Sevestre and Trognon (1983), Chamberlain (1984) and Mutl (2006), we can write:

$$
E^{*}\left[\mu_{j} \mid y_{10}, \ldots, y_{N 0}\right]=\lambda^{\prime}\left[y_{0}-E\left[y_{0}\right]\right]
$$

with

$$
\begin{aligned}
y_{0} & =\frac{1}{1-\gamma} P_{N} \mu_{N}+\frac{1}{\sqrt{1-\gamma^{2}}} P_{N} v_{0} \\
V\left[y_{0}\right] & =\left(\frac{\sigma_{\mu}^{2}}{(1-\gamma)^{2}}+\frac{\sigma_{v}^{2}}{1-\gamma^{2}}\right) P_{N} P_{N}^{\prime} \\
E\left[\mu_{j} y_{0}\right] & =\frac{\sigma_{\mu}^{2}}{1-\gamma} P_{N} l_{j}
\end{aligned}
$$

where $P_{N}=\left(B_{N} G_{N}\right)^{-1}$ and $l_{j}$ is the $j$ th column of $I_{N}$. 


\section{BIS Department tor Business

\section{Spatial Economics Research Centre (SERC)}

London School of Economics

Houghton Street

London WC2A 2AE

Tel: 02078523565

Fax: 02079556848

Web: www.spatialeconomics.ac.uk

SERC is an independent research centre funded by the Economic and Social Research Council (ESRC), Department for Business Innovation and Skills (BIS), the Department for Communities and Local Government (CLG) and the Welsh Assembly Government. 